\title{
Evaluation of two experimental models of hepatic encephalopathy in rats
}

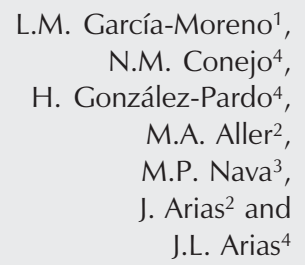

\author{
${ }^{1}$ Departamento de Psicobiologia, Facultad de Psicologia, \\ ${ }^{2}$ Departamento de Cirugía I, Facultad de Medicina, and \\ ${ }^{3}$ Departamento de Fisiologia (Fisiologia Animal II), \\ Facultad de Ciencias Biologicas, Universidade Complutense, \\ Madrid, España \\ ${ }^{4}$ Laboratorio de Psicobiologia, Facultad de Psicologia, \\ Universidade Oviedo, Asturias, España
}

\section{Correspondence}

M.A. Aller

Cátedra de Cirugía

Facultad de Medicina, U.C.M.

Plaza de Ramón y Cajal, s/n

28040 Madrid

Spain

Fax: +34-9-1394-7115

E-mail: maaller@med.ucm.es

Received January 22, 2004

Accepted October 27, 2004

\begin{abstract}
The serious neuropsychological repercussions of hepatic encephalopathy have led to the creation of several experimental models in order to better understand the pathogenesis of the disease. In the present investigation, two possible causes of hepatic encephalopathy, cholestasis and portal hypertension, were chosen to study the behavioral impairments caused by the disease using an object recognition task. This working memory test is based on a paradigm of spontaneous delayed non-matching to sample and was performed 60 days after surgery. Male Wistar rats (225-250 g) were divided into three groups: two experimental groups, microsurgical cholestasis $(\mathrm{N}=20)$ and extrahepatic portal hypertension $(\mathrm{N}=20)$, and a control group $(\mathrm{N}=$ 20). A mild alteration of the recognition memory occurred in rats with cholestasis compared to control rats and portal hypertensive rats. The latter group showed the poorest performance on the basis of the behavioral indexes tested. In particular, only the control group spent significantly more time exploring novel objects compared to familiar ones $(\mathrm{P}<0.001)$. In addition, the portal hypertension group spent the shortest time exploring both the novel and familiar objects $(\mathrm{P}<0.001)$. These results suggest that the existence of portosystemic collateral circulation per se may be responsible for subclinical encephalopathy.
\end{abstract}

\section{Introduction}

Hepatic encephalopathy (HE) is a neuropsychiatric syndrome observed in patients with liver failure and/or portal-systemic bypass. It is frequently considered to be a complex syndrome involving several behavioral manifestations such as personality changes, memory disorders, disorientation, flapping
Key words

- Cholestasis

- Portal hypertension

- Encephalopathy

- Behavior

- Recognition memory test ...... tremor, shortened attention span, lack of muscle coordination, bradykinesia, somnolence, and changes in sleep pattern (1). However, inconsistent results regarding the behavioral, metabolic and neurochemical characteristics indicate that the pathogenesis and pathophysiology of the syndrome are still obscure (2). Many investigators have reported that this could be caused by the lack of well- 
standardized animal models (3). Although the most extensively used experimental model of HE is the end-to-side portacaval anastomosis (4), new surgical techniques have been developed to overcome the reported limitations of this model (5). Therefore, we have selected two different experimental models which simulate several main pathological features that can cause HE associated with cirrhosis: portal hypertension (PHT) and cholestasis (CHO). Both models present two frequently associated main pathologic findings of chronic liver diseases, i.e., PHT and HE (6). PHT may be an early manifestation of cirrhosis. It is defined as an increase in hydrostatic pressure of the portal vein, commonly caused by a fibrotic obstruction of hepatic blood flow (7).

Although the pathophysiological consequences of PHT per se are still not well understood, a few behavioral manifestations have been reported, such as motor incoordination (8) and marked general intellectual impairment but preserved verbal ability (9). On the other hand, extrahepatic $\mathrm{CHO}$, which consists of bile stagnation in the liver, involves a combination of hepatic insufficiency and PHT. Its neurobehavioral outcomes have been partially investigated and some authors have reported clear neuropsychological impairments like fatigue and memory loss (10), together with anatomical changes in the central nervous system (CNS) (11). However, the behavioral impairments occurring in the two experimental models of PHT have not been compared. Thus, in the present study, we tested working memory ability in $\mathrm{CHO}$ and PHT rats using an original behavioral procedure that is highly sensitive to impairments of the hippocampal formation, a CNS structure directly related to learning and memory function in rats.

\section{Material and Methods}

Male Wistar rats (225-250 g) were housed in cages in temperature-controlled rooms and received water and food ad libitum. All animal treatments and surgical procedures employed in this study were performed in accordance with the principles and practices of the 1986 Guidelines for the Care and Use of Laboratory Animals (EC 86/609), published in Spain in RD 223/1988. Animals were randomly divided into three groups: a control group $(\mathrm{N}=20)$, a group of rats with microsurgical $\mathrm{CHO}(\mathrm{N}=20)$, which underwent a resection of the bile ducts that drain each hepatic lobe in continuity with the common bile duct (12). These rats showed increased hepatobiliary parameters and histological lesions which confirm CHO. And a third group of rats with extrahepatic PHT (N $=20$ ) induced by a triple portal vein ligation which causes a calibrated vascular stenosis (13) as assessed by the measurements of PHT.

The behavioral test was carried out 60 days after surgery in all groups. A spontaneous object recognition memory test was applied using a constantly illuminated wood open-field arena (dimensions: 65 x 45 x 45 $\mathrm{cm}$ ). The set of objects selected for visual discrimination was made of wood and plastic and objects were available in triplicate. The texture and shape of the objects were similar to those described by Ennaceur and Delacour (14). Initially, the animals were placed inside the box and were allowed to explore it for $3 \mathrm{~min}$ during habituation. Thereafter, each rat was tested in two different sessions separated by a 48 -h interval. Each session consisted of two different trials: in the first trial ( $\mathrm{T} 1$ or sample phase) two identical objects were placed in the corners of the box. In the second trial (T2 or test phase) the objects were replaced with a new set (novel and familiar objects). The position of the objects was randomly changed for each animal and session. The time allowed for exploration in each trial was $3 \mathrm{~min}$. The following variables were analyzed according to the procedure proposed by Ennaceur and Delacour (14): 1) Discrimination index: the 
difference between the time spent to explore the new object $(\mathrm{B})$ and the time spent to explore the familiar one (A) during the test phase (T2). 2) Index of habituation to the exploratory behavior defined as the total exploration time of the objects in the sample phase (T1) and test phase (T2). 3) Index of habituation to the sample: time spent to explore the familiar object (A) in the sample phase (T1) and the test phase (T2).

Data were analyzed statistically by oneway analysis of variance (ANOVA) with time of exploration as the measured variable and the experimental group as independent variable. Post hoc Newman-Keuls tests were applied. The results were considered to be significant when $\mathrm{P}<0.05$.

\section{Results}

There were significant group differences in the following behavioral indexes: discrimination between objects $\left(\mathrm{F}_{2,57}=26.5\right.$, $\mathrm{P}<0.001)$ and habituation to the sample $\left(\mathrm{F}_{2,57}=17.6, \mathrm{P}<0.001\right)$. Further analysis revealed that the control group was able to discriminate between the novel and familiar objects during the test phase compared to the $\mathrm{CHO}$ and PHT groups (Figure 1). Although no statistically significant differences were found between the control and $\mathrm{CHO}$ groups in the time spent exploring the familiar object, a significant decrease in the time spent exploring the novel object was found in the $\mathrm{CHO}$ group compared to the control group. On the other hand, no significant differences were found in the three groups regarding total time used for exploration during the sample and test phases $\left(\mathrm{F}_{2,57}=\right.$ $0.618, \mathrm{P}=0.543$ ), as shown in Figure 2 . Moreover, only control rats showed habituation to the familiar object during the test phase (Figure 3). Finally, the PHT group showed the poorest performance in all of the behavioral indexes measured, as evidenced by the limited time spent exploring the available objects.

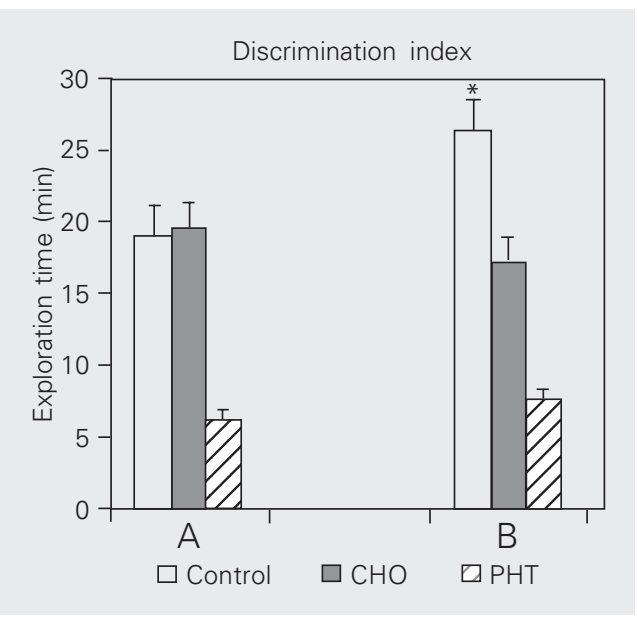

Figure 1. Exploration time for familiar (A) and novel (B) objects in the spontaneous object recognition test by rat models of hepatic hypertension. Control (N = 20); $\mathrm{CHO}=$ cholestasis $(\mathrm{N}=$ 20); $\mathrm{PHT}=$ portal hypertension $(\mathrm{N}=20) .{ }^{*} \mathrm{P}<0.05$ compared to object A (Newman-Keuls test).

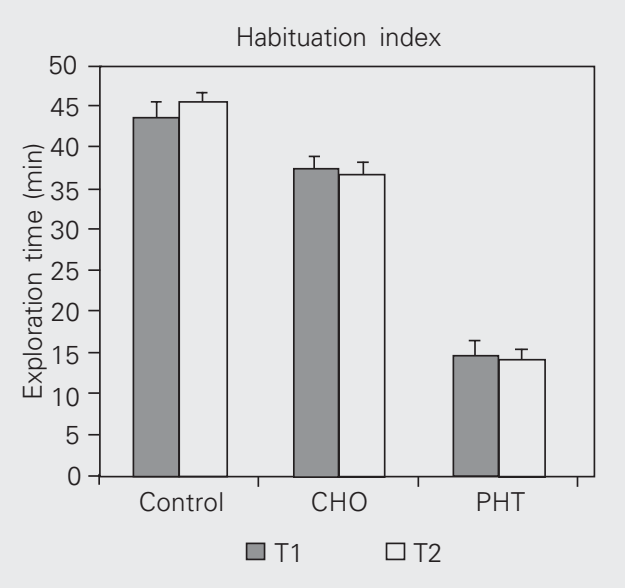

Figure 2. Index of habituation to the exploratory behavior. Control $(\mathrm{N}=20) ; \mathrm{CHO}=$ cholestasis $(\mathrm{N}=20)$; $\mathrm{PHT}=$ portal hypertension ( $N=20$ ). No significant differences were found between sample (T1) and test (T2) phases in any group.

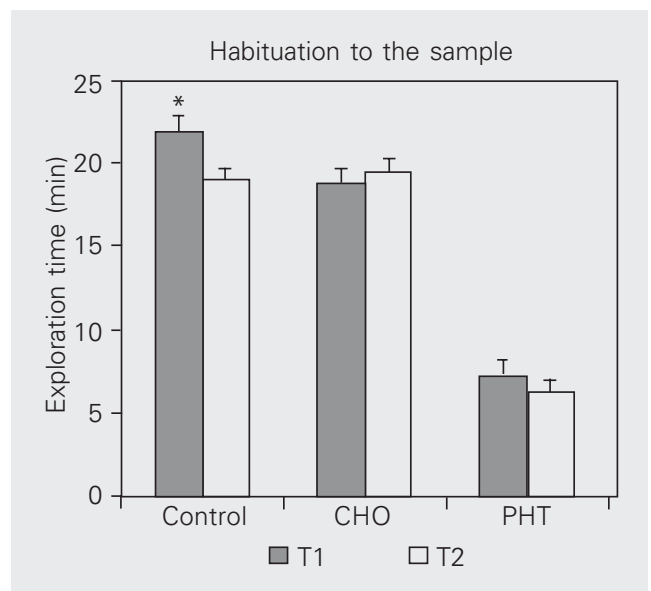

Figure 3. Index of habituation to the sample. Control ( $\mathrm{N}=20)$; $\mathrm{CHO}=$ cholestasis $(\mathrm{N}=20)$; $\mathrm{PHT}=$ portal hypertension $(\mathrm{N}=$ 20). $\mathrm{T} 1=$ sample phase; $\mathrm{T} 2=$ test phase. ${ }^{*} P<0.005$ compared to T2 (Newman-Keuls test). 


\section{Discussion}

Our results indicate that, unlike the control group, the $\mathrm{CHO}$ and PHT groups showed an impaired ability to discriminate between the novel object and the previously experienced sample object. In the literature, this novel object preference is termed "object recognition". However, a more serious memory impairment was observed in rats with $\mathrm{CHO}$ because they used excessive time to explore the familiar and novel objects, as shown in Figure 3. The animals with PHT, in turn, also displayed preference for the novel object although their exploration time was shorter than that spent by the control rats (Figure 3). The clear memory impairment of the $\mathrm{CHO}$ group indicates that PHT alone is not a sufficient condition to give rise to some of the main characteristics of HE.

The microsurgical extrahepatic $\mathrm{CHO}$ technique prevents both the formation of hilar biliary pseudocysts and of abdominalthoracic abscesses, complications which are common in macrosurgical $\mathrm{CHO}(15,16)$ and, therefore, reduces mortality compared to the bile duct ligation model (17). Since this microsurgical CHO model does not evolve with infection, it would be more appropriate than the macrosurgical technique of common bile duct ligation and section to study hepatic insufficiency and its consequences, among them, HE.

Thus, endotoxemia may be involved in the $\mathrm{HE}$ associated with $\mathrm{CHO}$ in addition to other factors such as hyperammonemia. Liver endotoxemia can stimulate Kupffer's cells to produce inflammatory cytokines such as tumor necrosis factor- $\alpha$, interleukin- 6 , and interleukin-1ß. These pro-inflammatory cytokines produced at the periphery can function as hormones and, since they can act in the CNS, they could produce HE.

It is clear from the results obtained in the present study that $\mathrm{CHO}$-induced encephalopathy represents a useful experimental model to simulate some of the neuropsycho- logical consequences of HE. In the first place, a similar impairment in working memory has been reported in patients with HE (18). In the second place, the time interval chosen (60 min) seems to involve the activation of hippocampal formation and not the prefrontal cortex (19). The hippocampus has been extensively related to recognition memory in rats $(20)$ or its equivalent in humans $(21,22)$. Recent data support the view that a reduction in long-term potentiation, the neural mechanism of memory found mainly in the hippocampus, could be responsible for the intellectual impairment reported in HE (23). This mechanism would favor the hyperammonemia hypothesis of HE since impairments in conditional discrimination behavior have also been reported in an animal model of hyperammonemia (24). Therefore, the working memory impairment induced by $\mathrm{CHO}$ could be caused indirectly by hyperammonemia, a common consequence of $\mathrm{CHO}$ (25). Although the molecular mechanisms of the role of ammonia in the neurological damage found in HE are still being discussed, the present study provides support for the ammonia hypothesis of HE and validates the $\mathrm{CHO}$ animal model of HE.

The etiology of HE arises from the gut, where toxic substances derived from food, drugs and bacterial metabolism are not detoxified by the liver, but are directly exposed to the brain causing cerebral disturbances. The etiology of HE, therefore, is probably multifactorial, with elevated blood ammonia levels playing an important and central role (26).

One of the mechanisms of ammonia detoxification in the liver is the urea cycle. The brain does not have a urea cycle and metabolizes ammonia to glutamine, causing a reduction in the intracerebral levels of glutamate, which is one of the most important excitatory neurotransmitters. The ammonia toxicity is probably more complex because, among other things, it also stimulates nitric oxide synthase causing cere- 
brovascular disorders, and benzodiazepine receptors, which stimulate the GABA receptor (the most important neurotransmission inhibiting receptor) by generating neurosteroids (26). Current evidence suggests that neurotransmission changes rather than brain energy failure are the primary cause of HE. Liver failure results in altered expression of several genes coding for proteins having key roles in the control of neuronal excitability. Such alterations include decreased expression of the glutamate transporter-1, and increased expression of monoamine oxidaseA isoform, the "peripheral-type" benzodiazepine receptor, as well as of constitutive neuronal nitric oxide synthase. These changes result in altered protein expression and in increased extracellular brain glutamate, increased degradation of monoamine neurotransmitters, increased synthesis of neurosteroids with inhibitory properties, and increased production of nitric oxide in the brain in chronic liver disease $(27,28)$. Prehepatic PHT by partial portal vein ligation leads to morphologic changes in the rat's CNS, including alterations of the blood brain barrier, astrogliosis and angiogenesis in the hippocampus, as well as functional changes in the integrity of the blood brain barrier and subtle impairments in brain activity which manifest as behavioral alterations such as motor incoordination. Thus, in the present study, we observed that the time of object exploration was reduced in rats with PHT. However, these rats tended to interact more with a familiar object in a free-choice situation, suggesting the development of a subclinical form of HE (29).

The experimental models of microsurgical CHO and PHT share intestinal bacterial translocation, development of portosystemic collateral circulation, and increased hypothalamic levels of leu-enkephalin (30), although these alterations are more intense in CHO. This could be the reason why HE, assessed in this study by memory impairment, was also more intense in $\mathrm{CHO}$ while it was occasionally subclinical in rats with PHT, producing a mild alteration in the object recognition task.

A new nomenclature of $\mathrm{HE}$ has been used since 2002, classifying HE into encephalopathy type A, associated with acute liver failure, type B, associated with portal systemic bypass without intrinsic hepatocellular disease, and type $\mathrm{C}$, associated with liver cirrhosis (31). According to this new classification, CHO-induced $\mathrm{HE}$ would correspond to type $\mathrm{C}$ whereas $\mathrm{PHT}$, in which there is only portal-systemic circulation without hepatic insufficiency, would occur with subclinical HE named type B in the human clinical area.

\section{References}

1. Butterworth RF (1996). The neurobiology of hepatic encephalopathy. Seminars in Liver Disease, 16: 235-244.

2. Albrecht J \& Jones EA (1999). Hepatic encephalopathy: molecular mechanisms underlying the clinical syndrome. Journal of the Neurological Sciences, 170: 138-146.

3. Mullen KD \& McCullough AJ (1989). Problems with animal models of chronic liver disease: suggestions for improvement in standardization. Hepatology, 9: 500-503.

4. Lee SH \& Fisher B (1961). Portocaval shunt in the rat. Surgery, 50: 668-672.

5. Chamuleau RA (1996). Animal models of hepatic encephalopathy. Seminars in Liver Disease, 16: 265-271.

6. Sherlock S (2002). The portal venous system and portal hypertension. In: Sherlock S (Editor), Diseases of the Liver and Biliary Sys- tem. 11th edn. Blackwell Scientific Publications, Oxford, UK, 151207.

7. Menon KV \& Kamath PS (2000). Managing the complications of cirrhosis. Mayo Clinic Proceedings, 75: 501-509.

8. Schomerus H \& Hamster W (1998). Neuropsychological aspects of portal-systemic encephalopathy. Metabolic Brain Disease, 12: 361367.

9. Rehnstrom S, Simert G, Hansson JA, Johnson G \& Vang J (1977). Chronic hepatic encephalopathy. A psychometrical study. Scandinavian Journal of Gastroenterology, 12: 305-311.

10. Floreani $A$, Marchiori $M$, Bonato $S$, Zucchetto $M$, Naccarato $R$ \& Chiaramonte M (1995). Cognitive assessment in primary biliary cirrhosis: a case-control study. American Journal of Gastroenterology, 90: 250-253. 
11. Bernthal P, Hays A, Tarter RE, Van Thiel D, Lecky J \& Hegedus A (1987). Cerebral CT scan abnormalities in cholestatic and hepatocellular disease and their relationship to neuropsychologic test performance. Hepatology, 7: 107-114.

12. López L, Begega A, Arias JL, Lorente L, Aller MA, Durán M \& Arias $J$ (1999). Hepatic cytochrome oxidase in rats with microsurgical cholestasis or portocaval shunt. Revista Española de Enfermedades Digestivas, 91: 365-373.

13. Rodríguez G, Monterde G, Dieguez B, Aller MA \& Arias J (2000) Hipertensión portal a largo plazo en la rata por triple ligadura estenosante de la vena porta. Anales de Medicina Interna, 17: 137141.

14. Ennaceur A \& Delacour JA (1988). A new one-trial test for neurobiological studies of memory in rats. I. Behavioral data. Behavioural Brain Research, 21: 47-59.

15. Aller MA, Lorente $L$ \& Arias J (1991). Hepatic artery ischaemia in rats with cholestasis. Different response of the lobes. Journal of Hepatology, 13 (Suppl II): S87 (Abstract).

16. Aller MA, Lorente L, Alonso S, Rodríguez G \& Arias J (1993). Different types of response to hepatic arterial ischemia in rats with cholestasis. Surgical Research Communications, 14: 269-280.

17. Aller MA, Lorente L, Alonso S \& Arias J (1993). A model of cholestasis in the rat, using a microsurgical technique. Scandinavian Journal of Gastroenterology, 28: 10-14.

18. Elithorn A, Lunzer M \& Weinman J (1975). Cognitive deficits associated with chronic hepatic encephalopathy and their response to levodopa. Journal of Neurology, Neurosurgery and Psychiatry, 38: 794-798.

19. Ennaceur A \& Meliani K (1992). A new one-trial test for neurobiological studies of memory in rats. III. Spatial vs non-spatial working memory. Behavioural Brain Research, 51: 83-92.

20. Steele K \& Rawlins JN (1993). The effects of hippocampectomy on performance by rats of a running recognition task using long lists of non-spatial items. Behavioural Brain Research, 54: 1-10.

21. Desgranges B, Baron JC \& Eustache $F$ (1998). The functional neuroanatomy of episodic memory: the role of the frontal lobes, the hippocampal formation, and other areas. Neuroimage, 8: 198-210.

22. Stern CE, Corkin S, González RG, Guimaraes AR, Baker JR, Jennings PJ, Carr CA, Sugiura RM, Vedantham V \& Rosen BR (1996). The hippocampal formation participates in novel picture encoding: evidence from functional magnetic resonance imaging. Proceedings of the National Academy of Sciences, USA, 93: 8660-8665.

23. Muñoz MD, Monfort P, Gaztelu JM \& Felipo V (2000). Hyperammonemia impairs NMDA receptor-dependent long-term potentiation in the CA1 of rat hippocampus in vitro. Neurochemical Research, 25: 437-441.

24. Aguilar MA, Miñarro J \& Felipo V (2000). Chronic moderate hyperammonemia impairs active and passive avoidance behavior and conditional discrimination learning in rats. Experimental Neurology, 161: 704-713.

25. Koyama K, Owada $Y$ \& Yamauchi H (1979). Ammonia metabolism in the rat liver in biliary obstruction. Journal of Experimental Medicine, 127: 379-387.

26. Quero JC, Carmona I, García JM, Jiménez M \& Herrerías JM (2003) La encefalopatía hepática: terminología, etiología y tratamiento. Revista Española de Enfermedades Digestivas, 95: 127-134.

27. Butterworth RF (1998). Alterations of neurotransmitter-related gene expression in human and experimental portal-systemic encephalopathy. Metabolic Brain Disease, 13: 337-349.

28. Butterworth RF (2001). Neurotransmitter dysfunction in hepatic encephalopathy: new approaches and new findings. Metabolic Brain Disease, 16: 55-65

29. Scorticati C, Prestifilippo JP, Murer G, Lemberg A \& Perazzo JC (2001). Cambios funcionales en el sistema nervioso central de ratas hipertensas portales prehepáticas. Medicina, 61: 673-675.

30. Klin MJ, Waluga M, Madej A, Plewka A \& Herman ZS (1993). Hypothalamic, striatal and plasma leucine enkephalin level in two rat models of portal hypertension. Polish Journal of Pharmacology, 45: 281-290.

31. Ferenci $P$, Lockwood A, Mullen $K$, Tarter $R$, Weissenborn $K$ \& Blei AT (2002). Hepatic encephalopathy - Definition, nomenclature, diagnosis and quantification. Hepatology, 35: 716-721. 